\title{
Optical anisotropy induced by torsion stresses in the crystals belonging to point symmetry groups 3 and $\overline{3}$
}

\author{
Skab I. \\ Institute of Physical Optics, 23 Dragomanov St., 79005 Lviv, Ukraine, \\ e-mail: skab@ifo.lviv.ua
}

Received: 27.06 .2012

\begin{abstract}
The torsion method for measuring piezooptic coefficients $\pi_{14}$ and $\pi_{25}$ in the trigonal crystals belonging to the point symmetry groups 3 and $\overline{3}$ is described. The $X Y$-distributions of the optical indicatrix orientation and the optical birefringence induced by the torsion stresses are analysed and evaluated quantitatively. It is shown that the spatial birefringence distribution in the sample subjected to torsion around the $Z$ axis represents a canonical cone. The combined piezooptic coefficient for lead germanate crystals is determined experimentally. It is shown that, for all of the point symmetry groups of trigonal system, the torsion torque applied around the directions of optic axes would lead to appearance of a purely screw dislocation of the phase front of the outgoing wave, while the corresponding optical vortex would possess a unit topological charge.
\end{abstract}

Keywords: trigonal crystals, piezooptic effect, torsion stresses, optical vortices

PACS: $78.20 . \mathrm{Hp}, 78.20 . \mathrm{Ci}$

UDC: 535.55

\section{Introduction}

In our recent works [1-6] we have demonstrated that the piezooptic coefficients associated with shear mechanical stresses can be determined with high enough accuracy, when applying torsion stresses to crystal samples. In particular, we have determined the piezooptic coefficient $\pi_{14}$ for $\mathrm{LiNbO}_{3}$ (the point symmetry group $3 \mathrm{~m}$ ) and $\alpha-\mathrm{BaB}_{2} \mathrm{O}_{4}$ (the symmetry group $\overline{3} \mathrm{~m}$ ) crystals [2-4]. In so doing, we have reached the relative measurement accuracy not worse than 3.1 and $8.9 \%$, respectively for $\mathrm{LiNbO}_{3}$ and $\alpha-\mathrm{BaB}_{2} \mathrm{O}_{4}$. It is worthwhile to notice that the common techniques for measuring piezooptic coefficients usually yield much higher errors (see [7-9]).

In the frame of the method for measurements of piezooptic coefficients described in Ref. [3], one should apply torsion loads to crystals. Then the polarisation measurements often require a number of additional experimental conditions to be fulfilled. Namely, one has to scan the optical beam along the direction of one of eigen vectors of the optical impermeability tensor. In particular, the coefficient $\pi_{25}$ for the crystals belonging to the point symmetry groups 3 and $\overline{3}$ may be determined under a torsion torque applied around the $X$ axis and propagation of light in the same direction (the experimental geometry concisely denoted as $M_{x}, k \| X$ ). This should be done basing on experimental dependences of the induced birefringence $\delta(\Delta n)_{23}$ on the coordinate $Y$ at the condition $Z=0$ (i.e., the optical beam is scanned along the $Y$ axis). On the other hand, the coefficient $\pi_{14}$ may be determined using a dependence of the induced birefringence $\delta(\Delta n)_{13}$ on the coordinate 
$X$ in the experimental geometry denoted as $M_{y}, k \| Y$ and under the condition $Z=0$. Hence, in order to determine the coefficients $\pi_{25}$ and $\pi_{14}$ for the case of symmetry groups 3 and $\overline{3}$, one should carry out two polarisation measurements using two different crystalline samples of $X$ - and $Y$-cuts, provided that the orientations of the axes $Y$ and $X$ are known in advance.

It is also known $[6,10,11]$ that the torsion loading of crystals that belong to the trigonal or cubic symmetry systems is accompanied by appearance of optical vortices, whenever crystalline sample is placed between crossed circular polarisers. In other words, if a nearly plane wave is incident, then the outgoing wave passed through the circular analyser would bear a doughnut mode, i.e. the output intensity would be equal to zero in the centre of beam cross section, and a bright ring would be observed around a dark central region.

In the present work we consider a possibility for determining the piezooptic coefficients $\pi_{25}$ and $\pi_{14}$ in the crystals of symmetry groups 3 and $\overline{3}$ under the conditions that the light beam propagates along the optic axis in the $Z$-cut crystals and the torsion moment is applied around the same direction. In addition, we will analyse the main features of optical vortices appearing in such crystals.

\section{Results and discussion}

Let us consider the matrix of piezooptic tensor for the crystals of symmetry groups 3 and $\overline{3}$ :

\begin{tabular}{c|cccccc} 
& $\sigma_{11}$ & $\sigma_{22}$ & $\sigma_{33}$ & $\sigma_{32}$ & $\sigma_{31}$ & $\sigma_{21}$ \\
\hline$\Delta B_{11}$ & $\pi_{11}$ & $\pi_{12}$ & $\pi_{13}$ & $\pi_{14}$ & $-\pi_{25}$ & $2 \pi_{62}$ \\
$\Delta B_{22}$ & $\pi_{12}$ & $\pi_{11}$ & $\pi_{13}$ & $-\pi_{14}$ & $\pi_{25}$ & $-2 \pi_{62}$ \\
$\Delta B_{33}$ & $\pi_{31}$ & $\pi_{31}$ & $\pi_{33}$ & 0 & 0 & 0 \\
$\Delta B_{32}$ & $\pi_{41}$ & $-\pi_{41}$ & 0 & $\pi_{44}$ & $\pi_{45}$ & $2 \pi_{52}$ \\
$\Delta B_{31}$ & $-\pi_{52}$ & $\pi_{52}$ & 0 & $-\pi_{45}$ & $\pi_{44}$ & $2 \pi_{41}$ \\
$\Delta B_{21}$ & $-\pi_{62}$ & $\pi_{62}$ & 0 & $\pi_{25}$ & $\pi_{14}$ & $\pi_{66}=\pi_{11}-\pi_{12}$
\end{tabular}.

When a torsion moment $M_{z}$ is applied around the $Z$ axis to a crystalline sample of cylindrical shape, the two shear components of the mechanical stress tensor appear which may be written as [12]

$$
\sigma_{4}=\sigma_{32}=\frac{2 M_{z}}{\pi R^{4}} X
$$

and

$$
\sigma_{5}=\sigma_{31}=\frac{2 M_{z}}{\pi R^{4}} Y
$$

Here $R$ is the radius of cylindrical sample, whereas $X, Y$ and $Z$ are the principal axes of the Fresnel ellipsoid. The equation of optical indicatrix perturbed by the two shear stresses reads as

$$
\begin{aligned}
& \left(B_{11}+\pi_{14} \sigma_{4}-\pi_{25} \sigma_{5}\right) X^{2}+\left(B_{11}-\pi_{14} \sigma_{4}+\pi_{25} \sigma_{5}\right) Y^{2}+B_{33} Z^{2} \\
& +2\left(\pi_{44} \sigma_{4}+\pi_{45} \sigma_{5}\right) Y Z+2\left(\pi_{44} \sigma_{5}-\pi_{45} \sigma_{4}\right) X Z+2\left(\pi_{14} \sigma_{5}+\pi_{25} \sigma_{4}\right) X Y=1
\end{aligned},
$$

and the refractive indices in the $X Y$-cross section of the optical indicatrix are as follows:

$$
n_{1}=n_{o}-\frac{n_{o}^{3}}{2} \sqrt{\left(\pi_{14}^{2}+\pi_{25}^{2}\right)\left(\sigma_{4}^{2}+\sigma_{5}^{2}\right)}=n_{o}-n_{o}^{3} \frac{M_{z}}{\pi R^{4}} \sqrt{\left(\pi_{14}^{2}+\pi_{25}^{2}\right)\left(X^{2}+Y^{2}\right)}
$$




$$
n_{2}=n_{o}+\frac{n_{o}^{3}}{2} \sqrt{\left(\pi_{14}^{2}+\pi_{25}^{2}\right)\left(\sigma_{4}^{2}+\sigma_{5}^{2}\right)}=n_{o}+n_{o}^{3} \frac{M_{z}}{\pi R^{4}} \sqrt{\left(\pi_{14}^{2}+\pi_{25}^{2}\right)\left(X^{2}+Y^{2}\right)}
$$

As a result, the spatial distribution of the induced birefringence in the $X Y$-plane is given by the relation

$$
\Delta n_{12}=n_{o}^{3} \sqrt{\left(\pi_{14}^{2}+\pi_{25}^{2}\right)\left(\sigma_{4}^{2}+\sigma_{5}^{2}\right)}=2 n_{o}^{3} \frac{M_{z}}{\pi R^{4}} \sqrt{\left(\pi_{14}^{2}+\pi_{25}^{2}\right)\left(X^{2}+Y^{2}\right)},
$$

and the angle of optical indicatrix rotation around the $Z$ axis may be presented as

$$
\tan 2 \zeta_{Z}=\frac{\pi_{25} \sigma_{4}+\pi_{14} \sigma_{5}}{\pi_{14} \sigma_{4}-\pi_{25} \sigma_{5}}=\frac{\pi_{25} X+\pi_{14} Y}{\pi_{14} X-\pi_{25} Y} .
$$

Similar relations for the induced birefringence and the angle of optical indicatrix rotation have already been obtained in the recent works by the present authors $[5,11]$. However, an error has crept into the corresponding derivation when considering the form of the piezooptic tensor. Namely, the piezooptic coefficient $\pi_{15}$ has been taken as positive rather than negative, thus leading to incorrect conclusion about a possibility for appearance of elliptical vortices in the crystals of symmetry groups 3 and $\overline{3}$ subjected to torsion. In the present study we eliminate this inaccuracy.

Introducing the polar coordinate system $X=\rho \cos \varphi, Y=\rho \sin \varphi$, one can rewrite Eqs. (7) and (8) as

$$
\Delta n_{12}=2 n_{o}^{3} \frac{M_{z}}{\pi R^{4}} \sqrt{\left(\pi_{14}^{2}+\pi_{25}^{2}\right)\left(X^{2}+Y^{2}\right)}=2 n_{o}^{3} \frac{M_{z}}{\pi R^{4}} \rho \sqrt{\pi_{14}^{2}+\pi_{25}^{2}}
$$

and

$$
\tan 2 \zeta_{Z}=\frac{\pi_{25} X+\pi_{14} Y}{\pi_{14} X-\pi_{25} Y}=\frac{\pi_{25} \cos \varphi+\pi_{14} \sin \varphi}{\pi_{14} \cos \varphi-\pi_{25} \sin \varphi} .
$$

By solving the system of Eqs. (9) and (10), one can obtain the relations for the piezooptic coefficients $\pi_{14}$ and $\pi_{25}$ :

$$
\begin{aligned}
\pi_{14} & =\frac{\pi R^{4}\left(X \cos 2 \zeta_{Z}+Y \sin 2 \zeta_{Z}\right)}{2 n_{o}^{3} M_{z}\left(X^{2}+Y^{2}\right)} \Delta n_{12} \\
& =\frac{\pi R^{4}\left(\cos \varphi \cos 2 \zeta_{Z}+\sin \varphi \sin 2 \zeta_{Z}\right)}{2 n_{o}^{3} \rho M_{z}} \Delta n_{12}=\frac{\pi R^{4} \cos \left(\varphi-2 \zeta_{Z}\right)}{2 n_{o}^{3} \rho M_{z}} \Delta n_{12}, \\
\pi_{25} & =\frac{\pi R^{4}\left(X \sin 2 \zeta_{Z}-Y \cos 2 \zeta_{Z}\right)}{2 n_{o}^{3} M_{z}\left(X^{2}+Y^{2}\right)} \Delta n_{12} \\
& =\frac{\pi R^{4}\left(\cos \varphi \sin 2 \zeta_{Z}-\sin \varphi \cos 2 \zeta_{Z}\right)}{2 n_{o}^{3} \rho M_{z}} \Delta n_{12}=\frac{\pi R^{4} \sin \left(2 \zeta_{Z}-\varphi\right)}{2 n_{o}^{3} \rho M_{z}} \Delta n_{12}
\end{aligned}
$$

Thereby, one can accurately determine the piezooptic coefficients $\pi_{14}$ and $\pi_{25}$ using the experimental data on the $X Y$-distributions of the induced birefringence and the optical indicatrix rotation angle, together with Eqs. (11) and (12). The estimated error of determination of the both coefficients is close to that typical for the piezooptic coefficient $\pi_{14}$ measured for the case of lithium niobate crystals [2].

For further analysis let us introduce a parameter $K$ that determines the ratio of the piezooptic coefficients, $K=\pi_{25} / \pi_{14}=\tan 2 \varphi_{0}$. Then one can simplify Eq. (10) as 


$$
\tan 2 \zeta_{Z}=\tan \left(2 \varphi_{0}+\varphi\right), \quad \zeta_{Z}=\varphi_{0}+\frac{\varphi}{2}=\frac{1}{2} \arctan \left(\pi_{25} / \pi_{14}\right)+\frac{\varphi}{2},
$$

where $\varphi_{0}=\frac{1}{2} \arctan \left(\pi_{25} / \pi_{14}\right)$ is the initial optical indicatrix rotation angle.

It is evident from Eqs. (9) and (13) that the induced birefringence has a conical spatial distribution and so it would depend only on the distance from the centre of the $X Y$-cross section. On the other hand, the optical indicatrix rotation angle $\zeta_{Z}$ is determined by the polar angle $\varphi$ so that the angle $\zeta_{z}$ would change two times slower than the $\varphi$ value, starting from some initial value $\varphi_{0}$ that depends on the ratio of piezooptic coefficients.

As follows from Eq. (13), we have

$$
\tan 2 \zeta_{Z}=-\frac{\pi_{14}}{\pi_{25}}=-\frac{1}{K}, \quad \zeta_{Z}=\frac{1}{2} \arctan (-1 / K)=\frac{\pi}{4}+\varphi_{0}
$$

at $X=0$ and

$$
\tan 2 \zeta_{Z}=\frac{\pi_{25}}{\pi_{14}}=K, \quad \zeta_{Z}=\frac{1}{2} \arctan (K)=\varphi_{0}
$$

at $Y=0$. Thus, the optical indicatrix rotation angle under the condition $Y=0$ is determined by the piezooptic ratio $K$. A similar conclusion has earlier been drawn in our work [13] when analysing a torsion method for orientation of crystals belonging to the middle-system classes. As seen from Eq. (10), a zero optical indicatrix rotation $\zeta_{Z}$ is reached when $\pi_{25} \cos \varphi+\pi_{14} \sin \varphi=0$, i.e. at the polar angle $\varphi=\arctan \left(-\pi_{25} / \pi_{14}\right)=\arctan (-K)$. The dependences of the optical indicatrix rotation angle upon the polar angle calculated for different $K$ values are shown in Fig. 1. Fig. 1 testifies that a gradual change in the $K$ parameter leads to parallel displacement of the linear $\zeta_{Z}(\varphi)$ dependences, i.e. to change in the initial optical indicatrix rotation angle beginning from $0^{\circ}$ (for the case of $K=0$, i.e. $\pi_{25} \rightarrow 0$ ) up to the value $45^{\circ}$ (at $K=100$, i.e. $\pi_{14} \rightarrow 0$ ).

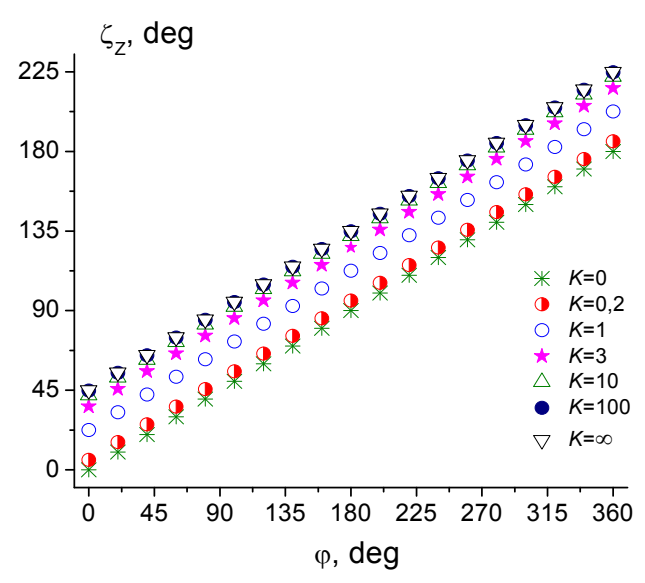

Fig. 1. Dependences of optical indicatrix rotation angle for the crystals of point symmetry groups 3 and $\overline{3}$ on the polar angle, calculated for different ratios $K=\pi_{25} / \pi_{14}$.

Ukr. J. Phys. Opt. 2012, V13, №3 
The spatial distributions of the optical indicatrix rotation angle in the $X Y$-plane calculated for the crystals belonging to the symmetry groups 3 and $\overline{3}$ are presented in Fig. 2 . As seen from Fig. 2, the angle of optical indicatrix rotation at $K=0$ starts from a zero value and increases linearly up to $180^{\circ}$ with increasing angle $\varphi$. However, the starting value of the optical indicatrix rotation at $K=1$ is equal to $22.5^{\circ}$ and the $\zeta_{Z}$ value reaches $202.5^{\circ}$ at $\varphi=360^{\circ}$. Finally, the value of $\zeta_{Z}$ is equal to $45^{\circ}$ and $225^{\circ}$ if $K=100$.
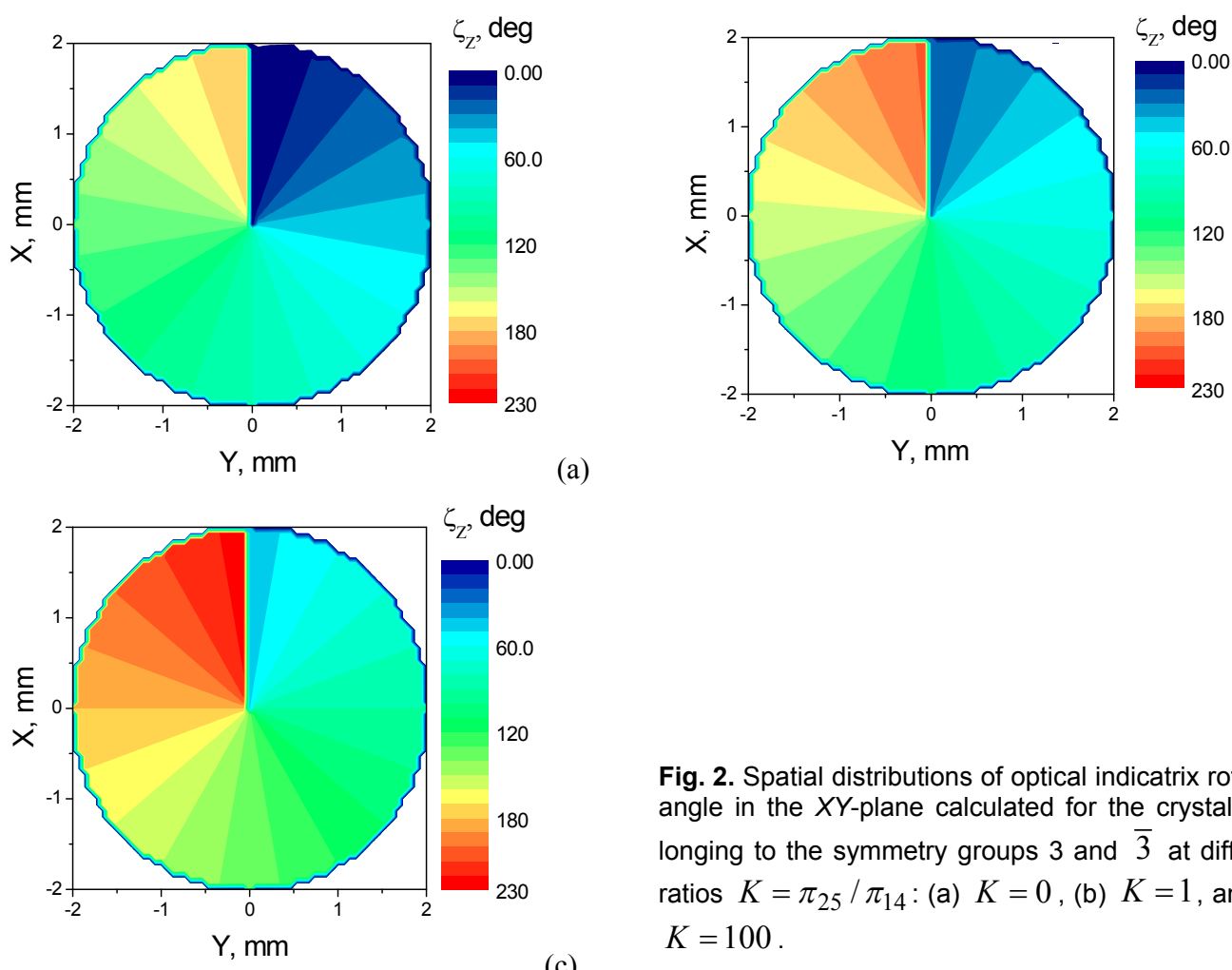

(c)

Fig. 2. Spatial distributions of optical indicatrix rotation angle in the $X Y$-plane calculated for the crystals belonging to the symmetry groups 3 and $\overline{3}$ at different ratios $K=\pi_{25} / \pi_{14}:$ (a) $K=0$, (b) $K=1$, and (c) $K=100$.

Now let us analyse the spatial distribution of the birefringence induced in the $X Y$-plane by the torsion moment $M_{Z}=0.049 \mathrm{~N} \times \mathrm{m}$ (the rest of the parameters are taken to be $n_{o}=2.0$, $R=2 \mathrm{~mm}$, and $\left.\pi_{14} \approx 10^{-12} \mathrm{~m}^{2} / \mathrm{N}\right)$. As follows from Eq. (9), this distribution has the shape of a canonical cone, with a zero induced birefringence in the centre of the $X Y$-cross-section of a twisted crystal (see Fig. 3). The induced birefringence does not change its sign at this point. The induced birefringence can reach the value as large as $\sim 10^{-5}$ for the parameters mentioned above.

As seen from Fig. 2 and Fig. 3, the $X Y$-plane distributions of the induced birefringence and the optical indicatrix orientation angle typical for the crystals belonging to the symmetry groups 3 and $\overline{3}$ are similar to those for the groups $32,3 m$ and $\overline{3} m$ [11], for which $\pi_{25}=0$. The former distributions differ only by a presence of nonzero initial angle of optical indicatrix rotation. Using these distributions and a standard Jones matrix approach [10], we have analysed the $X Y$-distribution of the optical phase retardation between the eigenwaves. Similarly to the case of lithium niobate crystals, variation of the angle $\varphi$ around the geometrical centre of this distribution changes the phase of optical wave by the same angle $[14,15]$. Hence, the spatial map of phase of the optical wave, which is undefined at the centre, can be represented as a canonical helicoid. 
Since the phase retardation angle coincides with the tracing angle, the vortex induced by the torsion should possess a topological charge modulo equal to unity, whereas the sign of the vortex charge is defined by the sign of the incident circularly polarised wave.
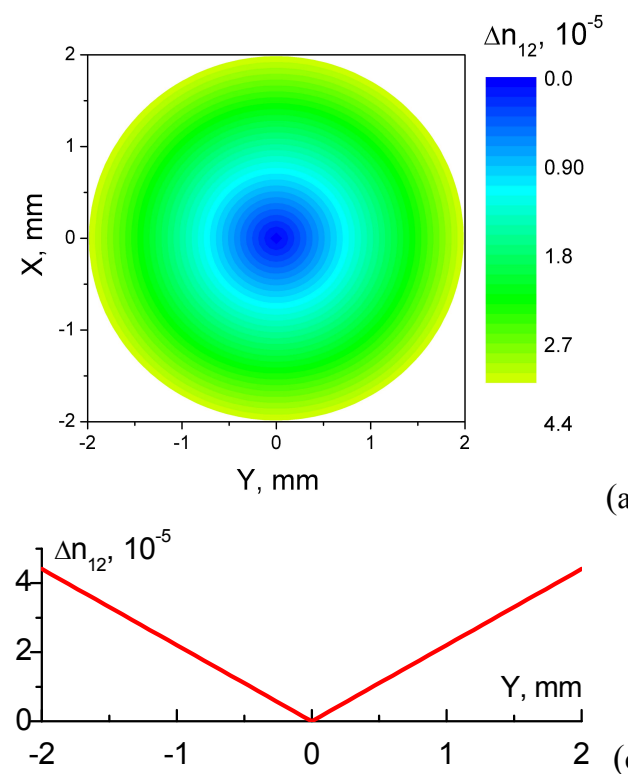

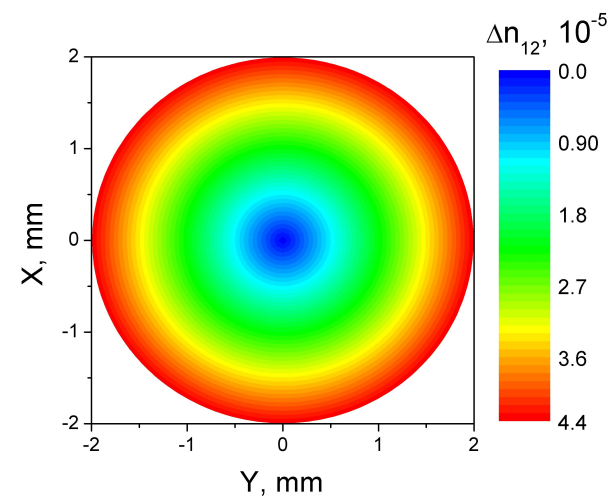

(b)

Fig. 3. Spatial distributions of birefringence in the $X Y$-plane $(a, b)$ and dependence of birefringence on the $Y$ coordinate at $X=0$ (c), which are induced by the torsion moment $M_{Z}=0.049 \mathrm{~N} \times \mathrm{m}$ in the crystals belonging to the point groups 3 and $\overline{3}:$ (a) $K=0$ and (c) (b, c) $K=1$.

Following from the above analysis, as well as the results reported in our recent works [10, 11], one can conclude that the torsion torque applied around the directions of their optic axes causes a purely screw dislocation of the phase front of the outgoing wave, whenever the initial light is nearly a plane wave and has the circular polarisation. This remains true for all of the point symmetry groups of trigonal system (i.e., the groups $32,3 m, \overline{3} m, 3$, and $\overline{3}$ ). Then the output optical beam would carry the optical vortex with a topological charge equal to unity. The appearance of such an optical vortex has been observed by the authors under torsion of $\mathrm{Pb}_{5} \mathrm{Ge}_{3} \mathrm{O}_{11}$ crystals (the symmetry group 3) [6]. In the latter case a doughnut mode with a ring-shaped intensity distribution has been revealed for the $\mathrm{Pb}_{5} \mathrm{Ge}_{3} \mathrm{O}_{11}$ crystal twisted around the $Z$ axis, provided that the sample is placed in between right-handed and left-handed circular polarisers. This intensity distribution agrees well with our present analysis and indeed points to a presence of the purely screw dislocation in the wave front. Using the intensity distributions obtained experimentally in the work [6], one can determine the combined piezooptic coefficient for the lead germanate as $\sqrt{\pi_{14}^{2}+\pi_{25}^{2}}=(0.35 \pm 0.09) \times 10^{-12} \mathrm{~m}^{2} / \mathrm{N}$.

\section{Conclusion}

In the present work we have described a method for measuring piezooptic coefficients $\pi_{25}$ and $\pi_{14}$ for the trigonal crystals belonging to the point symmetry groups 3 and $\overline{3}$, and determined in this manner the combined piezooptic coefficient for the lead germanate crystals $\left(\sqrt{\pi_{14}^{2}+\pi_{25}^{2}}=(0.35 \pm 0.09) \times 10^{-12} \mathrm{~m}^{2} / \mathrm{N}\right)$. We have also shown that the torsion moment applied around the directions of optic axes in the crystals of all the point symmetry groups belonging to the trigonal system (i.e., for the crystals with the symmetries $32,3 m, \overline{3} m, 3$, and $\overline{3}$ ) should lead to appearance of a purely screw dislocation in the phase front of the outgoing wave, under the conditions that the incident light represents nearly a plane wave and is circularly polarised. As a result, the output optical beam should carry the optical vortex with the unit topological charge. 


\section{Acknowledgment}

The author acknowledge the State Fund for Fundamental Researches of Ukraine for financial support of present study (project F41.1/010).

\section{References}

1. Skab I, Vasylkiv Yu, Savaryn V and Vlokh R, 2010. Relations for optical indicatrix parameters in the conditions of crystal torsion. Ukr. J. Phys. Opt. 11: 193-240.

2. Vasylkiv Yu, Savaryn V, Smaga I, Skab I and Vokh R, 2010. Determination of piezooptic coefficient $\pi_{14}$ of $\mathrm{LiNbO}_{3}$ crystals under torsion loading. Ukr. J. Phys. Opt. 11: 156-164.

3. Skab I, Smaga I, Savaryn V, Vasylkiv Yu and Vlokh R, 2011. Torsion method for measuring piezooptic coefficients. Cryst. Res. \& Techn. 46: 23-36.

4. Vasylkiv Y, Savaryn V, Smaga I, Skab I and Vlokh R, 2011. On determination of sign of the piezooptic coefficients using torsion method. Appl. Opt. 50: 2512-2518.

5. Skab I, Vasylkiv Yu, Smaga I, Savaryn V and Vlokh R, 2011. On the method for measuring piezooptic coefficients $\pi_{25}$ and $\pi_{14}$ in the crystals belonging to point symmetry groups 3 and $\overline{3}$. Ukr. J. Phys. Opt. 12: 28-35.

6. Vasylkiv Yu, Skab I and Vlokh R, 2011. Measurements of piezooptic coefficients $\pi_{14}$ and $\pi_{25}$ in $\mathrm{Pb}_{5} \mathrm{Ge}_{3} \mathrm{O}_{11}$ crystals using torsion induced optical vortex. Ukr. J. Phys. Opt. 12: 101-108.

7. Vasylkiv Yu, Kvasnyuk O, Krupych O, Mys O, Maksymuk O and Vlokh R, 2009. Reconstruction of 3D stress fields basing on piezooptic experiment. Ukr. J. Phys. Opt. 10: 22-37.

8. Mytsyk B, 2003. Methods for the studies of the piezo-optical effect in crystals and the analysis of experimental data. I. Methodology for the studies of piezo-optical effect. Ukr. J. Phys. Opt. 4: 1-26.

9. Narasimhamurty T S, Photoelastic and electrooptic properties of crystals. New York: Plenum Press (1981).

10. Skab I, Vasylkiv Y, Savaryn V and Vlokh R, 2011. Optical anisotropy induced by torsion stresses in $\mathrm{LiNbO}_{3}$ crystals: appearance of an optical vortex. J. Opt. Soc. Amer. A. 28: 633640 .

11. Skab I, Vasylkiv Y, Zapeka B, Savaryn V and Vlokh R, 2011. On the appearance of singularities of optical field under torsion of crystals containing three-fold symmetry axes. J. Opt. Soc. Amer. A. 28: 1331-1340.

12. Sirotin Yu I and Shaskolskaya M P, Fundamentals of crystal physics. Moscow: Nauka (1979).

13. Vlokh R, Pyatak Y and Skab I, 1992. On the method for orientation of middle-system crystals. Ukr. Fiz. Zhurn. 37: 207-210.

14. Berry M V, 1984. Quantal phase factors accompanying adiabatic changes. Proc. Roy. Soc. Lond. A Mat. 392: 42-57.

15. Shapere A and Wilczek F, Geometric phases in physics. Singapore: World Scientific (1989).

Skab I., 2012. Optical anisotropy induced by torsion stresses in the crystals belonging to point symmetry groups 3 and $\overline{\mathbf{3}}$. Ukr.J.Phys.Opt. 13: $158-164$.

Анотація. У изій роботі описано торсійний метод вимірювання n'єзооптичних коефіцієнтів $\pi_{14} i \pi_{25}$ в тригональних кристалах, щзо належать до точкових груп симетрії $3 i \overline{3}$. Отримано та проаналізовано ХY-розподіли орієнтачії оптичних індикатрис і двозаломлення, індуковані торсійними напруженнями. Показано, щзо розподіл двозаломлення за умови кручення кристалів навколо осі $Z$ має вигляд канонічного конуса. Експериментально визначено комбінований n'єзооптичний коефіцієнт для кристалів германату свинцю. Показано, щзо для всіх точкових груп кристалів тригональної сингонії торсійний момент, прикладений навколо їхньої оптичної осі, приведе до появи чистої гвинтової дислокачії хвильового фронту і оптичного вихору з одиничним топологічним зарядом. 